ISSN electrónico: 2445-1355

DOI: https://doi.org/10.14201/fj2021612737

\title{
ESTUDIO DE ncRNA EN VARONES CON CÁNCER DE MAMA
}

\section{ncRNA Study in Males with Breast Cancer}

David DÍEZ CASTRO; María OVEJERO SÁNCHEZ; Rogelio GONZÁLEZ SARMIENTO

Unidad de Medicina Molecular. Departamento de Medicina. Facultad de Medicina. Universidad de Salamanca. Calle Alfonso X el Sabio, s/n. 37007 Salamanca

Correo-e: dadicas@usal.es

RESUMEN: El cáncer de mama (CM) en varones es una enfermedad rara, ya que se trata de menos del $1 \%$ de los cánceres de mama detectados. Por ello es importante encontrar formas de prevenir este tipo de enfermedades y saber por qué se producen y si existe algún factor genético que predispone a padecer este tipo de cáncer. En este sentido, se sabe que algunos RNA no codificantes (ncRNA) están involucrados en el CM en mujeres, pero no hay nada descrito referente a su implicación en varones. En este trabajo se pretende averiguar si determinados ncRNA predisponen o protegen a varones de padecer CM. En concreto, se han estudiado algunos polimorfismos de nucleótido único de los genes que codifican para los mir-146A, mir-196A2, mir-499A y el lnRNA HotAIR mediante la técnica de genotipado con sondas Taqman, que permite discriminar entre los polimorfismos de cada ncRNA. La población de estudio ha sido facilitada por el Consejo Genético del Centro de Investigación del Cáncer. Los resultados obtenidos indican que el alelo C del polimorfismo rs2910164 del gen que codifica el mir-146A y el genotipo CA del polimorfismo rs1899663 del gen que codifica HotAIR podrían proteger frente a la aparición de CM en varones.

Palabras clave: ncRNA; cáncer de mama en varones; miR-146A; miR-196A2; miR-499A; HotAIR. 
ABSTRACT: Male breast cancer (BC) is a rare disease accounting for less than $1 \%$ of all BC detected. For this reason, it would be important to find ways to prevent this kind of cancer, to know how it develops in men and if there is any genetic predisposition to have BC. We know that noncoding RNA (ncRNA) are involved in female breast cancer but there is no information about their role in males. In this paper we try to find if some ncRNA have implications in male BC. Specifically, we have studied some single nucleotide polymorphisms of the genes encoding for mir-146A, mir-196A2, mir-499A and long noncoding RNA, HotAIR. The technique used was genotyping with taqman probe, that allows to discriminate between different polymorphisms. The Genetic Counsil of Cancer Research Center provides us with the study population. The preliminary results showed that the allele C of the polymorphism with rs2910164 of the mir-146A gene and the genotype CA of the polymorphism with rs1899663 of the HotAIR gene could protect males from developing BC.

Keywords: ncRNA; Male Breast Cancer; miR-146A; miR-196A2; miR-499A; HotAIR.

\section{INTRODUCCIÓN}

El ácido ribonucleico no codificante (ncRNA) es un tipo de ácido ribonucleico (RNA) que no se traduce a proteína, las funciones que tienen estos RNA son muy variadas, y algunas muy importantes para la célula; entre ellas están el procesamiento y la regulación de otros RNA como el mensajero, el transferente y el ribosómico. Se pueden dividir en dos grupos, de cadena corta y de cadena larga (Jeffares et al., 1998; Collins et al., 2011).

\section{$1.1 n c R N A$ de cadena corta}

Normalmente tienen menos de 200 bases, este grupo se divide en tres tipos: micro RNA (miRNA), RNA interferente corto (siRNA) y RNA que interactúa con piwi (piRNA)(Collins et al., 2011). Este trabajo se centrará en el estudio de miRNA.

Los miRNA están formados de 22 bases aproximadamente, modulan la expresión génica de numerosos genes y de importantes funciones biológicas, como la diferenciación celular, la homeostasis tisular o la carcinogénesis. Además, estos miRNA pueden ser liberados al espacio extracelular y permanecen estables, lo que los hace accesibles en plasma u otros fluidos. Estos ncRNA se pueden 
medir fácilmente por técnicas como la reacción en cadena de la polimerasa (PCR) cuantitativa o el genotipado, por lo que se pueden usar como biomarcadores en algunas patologías como el cáncer. Algunos polimorfismos de nucleótido único (SNPs) en los genes que codifican miRNA se han asociado con un mayor riesgo a desarrollar cáncer (Collins et al., 2011; Peng y Croce, 2016):

- El polimorfismo rs2910164 del gen mir-146A es una sustitución de C por $\mathrm{G}$ que da lugar a una alteración en el bucle del miRNA. Un estudio in vivo muestra que el genotipo GG se asocia a una sobreexpresión del gen contribuyendo a una elevada proliferación de las células hepáticas (Zhang et al., 2016). Este miRNA está asociado a la actividad de BRCA1/BRCA2 (Upadhyaya et al., 2016).

- El polimorfismo rs11614913 del gen mir-196A2 es un cambio de C por T y puede tener un papel importante en la formación del cáncer de mama (CM). Según un estudio de $\mathrm{Hu}$ et al. (Hu et al., 2009), la población con los genotipos CC/CT es más propensa a padecer CM que aquella con el TT (Dai et al., 2016).

- El polimorfismo rs3746444 del gen mir-499A es la sustitución de una A por una $\mathrm{G}$, que influye en la regulación de la expresión de los genes FOX04, PDCD4 y SOX6, que son importantes en la etiología de los cánceres (Dai et al., 2016).

\section{$1.2 n c R N A$ de cadena larga}

Tienen más de 200 nucleótidos, forman la mayor parte del transcriptoma humano, la mayoría de ncRNA forman parte de este grupo. Los ncRNA largos (lncRNA) son capaces de formar complejos con proteínas modificadoras de cromatina, modificando así la cromatina y la transcripción génica (Figura 1). Entre sus funciones están la remodelación de la cromatina, la regulación transcripcional y postranscripcional, y funcionan como precursores de los siRNA (Collins et al., 2011; What is epigenetics, 2019).

Entre los lncRNA se encuentran los RNA intergénicos antisentido HOX (HotAIR), este tipo de lncRNA desempeñan un papel importante en el desarrollo del cáncer; entre sus funciones destacan la proliferación, supervivencia, migración celular, la resistencia a medicamentos y la estabilidad del genoma. Este tipo de ncRNA, al igual que los de cadena corta, se pueden usar como biomarcador en el cáncer (Tang y Hann, 2018). 


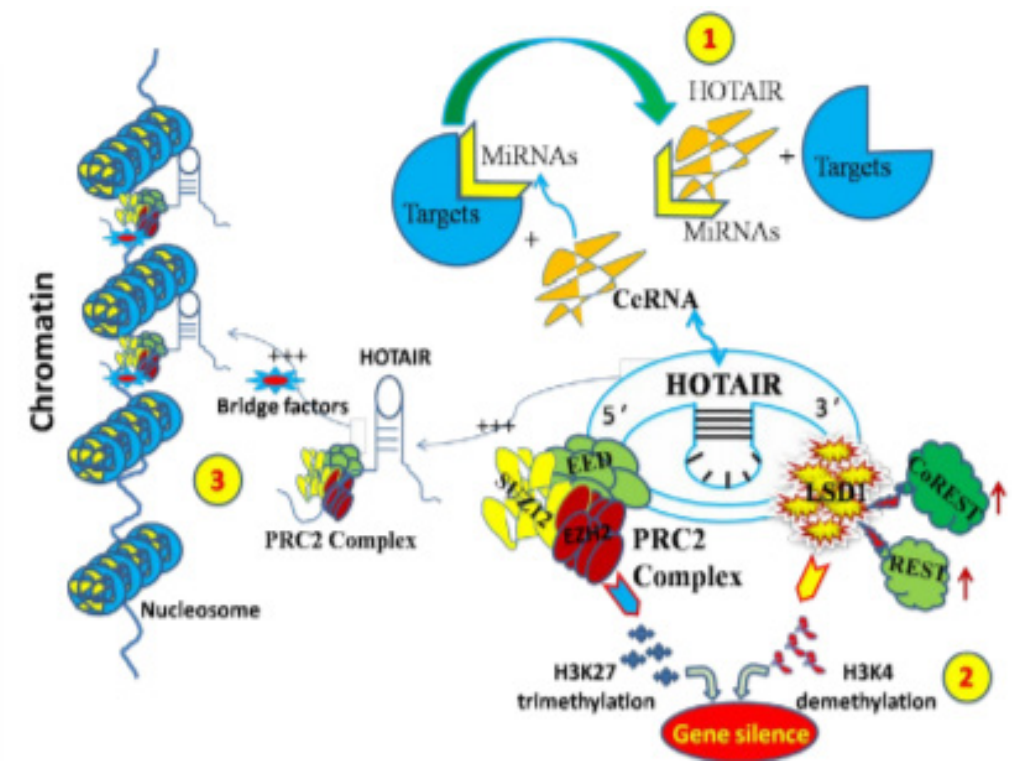

Figura 1. Mecanismo de acción del HOTAIR mediante la interacción con miRNA, silenciación de genes y modificación de la cromatina (Tang y Hann, 2018).

\section{Hipótesis y objetivos}

Teniendo en cuenta que los ncRNA son muy importantes en la regulación de la expresión génica y que la presencia de polimorfismos en los genes podría tener un papel importante en la función de estos, el objetivo de este estudio es determinar si la presencia de polimorfismos en los ncRNA (mir146A, mir196A2, mir499A y HotAir) influye en la predisposición a padecer $\mathrm{CM}$ en varones.

\section{Materiales y métodos}

\subsection{Población de estudio}

En este trabajo, se estudiaron 51 pacientes varones con $\mathrm{CM}$ y 74 controles varones sin CM. Las muestras fueron facilitadas por la unidad de Consejo Genético del Centro de Investigación del Cáncer (USAL-CSIC), en colaboración con el Complejo asistencial Universitario de Salamanca. 


\subsection{Extracción de DNA}

Realizamos la extracción de DNA a partir de células mononucleadas obtenidas de muestras de sangre periférica de la población incluida en el estudio mediante fenol-cloroformo (Banco de ADN, 2011).

Para obtener el DNA, se aislaron las células nucleadas de las muestras de sangre mediante centrifugación y, a continuación, se lisaron con un tampón FORNACE, EDTA, proteinasa $\mathrm{K}$ y SDS durante 16 horas a $55^{\circ} \mathrm{C}$. Una vez pasado este tiempo, se separaron todos los restos celulares del DNA gracias al método fenol-cloroformo, en el que el DNA queda en un solvente acuoso y los restos celulares en el orgánico. Por último, se precipitó con etanol y se eliminaron los restos del mismo por evaporación, tras lo cual se resuspendió en $\mathrm{ddH}_{2} \mathrm{O}$ y se almacenó a $-20^{\circ} \mathrm{C}$ hasta su uso.

\subsection{Genotipado}

La reacción en cadena de la polimerasa $(\mathrm{PCR})$ permite generar múltiples copias de un fragmento de DNA gracias a dos oligonucleótidos que son complementarios a los extremos del fragmento a amplificar y a una polimerasa que incorpora nucleótidos complementarios a la cadena molde. En la PCR a tiempo real, la amplificación y la detección se producen simultáneamente, ya que los componentes de la PCR emiten fluorescencia.

En este proceso se usan sondas TaqMan, cuyo funcionamiento se basa en la actividad de la enzima Taq polimerasa 5'-3'. En cada ensayo se añaden unos "primers», nucleótidos de alrededor de $20 \mathrm{pb}$, complementarios al fragmento de DNA que se quiere amplificar, y dos sondas TaqMan fluorescentes con un ligando de unión al surco menor del DNA. Cada sonda tiene unido un fluoróforo diferente, de forma covalente al extremo 5'. En el extremo 3' de la sonda hay un grupo que previene la liberación de la fluorescencia si la sonda no se degrada. Durante la reacción de la PCR las sondas hibridan con los fragmentos de DNA, en el polimorfismo que nos interesa y, cuando se amplifica el DNA, se destruyen liberando la fluorescencia que es lo que recoge el termociclador (Vieira, 2012; Patia, 2016).

En esta investigación se ha trabajado con 2 sondas, Vic y Fam. Cada una corresponde a uno de los alelos del polimorfismo, permitiendo discriminar entre los homocigóticos para Vic (Vic/Vic) y para Fam (Fam/Fam) y el heterocigótico (Vic/Fam).

En la Tabla 1 aparecen recogidos los genes que se han estudiado y su polimorfismo.

Ediciones Universidad de Salamanca / @@ $\quad$ FarmaJournal, vol. 6, núm. 1 (2021), pp. 27-37 
DAVID DÍEZ CASTRO; MARÍA OVEJERO SÁNCHEZ; ROGELIO GONZÁLEZ SARMIENTO ESTUDIO DE NCRNA EN VARONES CON CÁNCER DE MAMA

TABLA 1. Información de los genes estudiados

\begin{tabular}{c|cccc}
\hline \multicolumn{1}{c}{ GEN } & SNP ID & Cambio de base & Cromosoma & Sonda ID \\
\hline miR146A & rs2910164 & g.159912418C $>\mathrm{G}$ & 5 & C_15946974_10 \\
miR196A2 & rs11614913 & g.54385599C $>\mathrm{T}$ & 12 & C_31185852_10 \\
miR499A & rs3746444 & g.33578251A $>\mathrm{G}$ & 20 & C_2142612_30 \\
& rs1899663 & g.54360994C $>\mathrm{A}$ & 12 & C_2104251_20 \\
HOTAIR & rs12826786 & g.54355501C $>\mathrm{T}$ & 12 & C_31185830_10 \\
\cline { 2 - 6 } & & &
\end{tabular}

\subsection{Análisis estadístico}

El análisis estadístico se llevó a cabo con la prueba de $\mathrm{Chi}^{2}$ para determinar las diferencias entre la distribución genotípica y alélicas en los distintos grupos de estudio. Se tomaron como significativos los p-valores inferiores a 0,05, es decir, un intervalo de confianza del $95 \%$. Para llevar a cabo este estudio, se empleó el programa IBM SPSS Statistics 25.

\section{Resultados}

En este trabajo estudiamos 5 SNP de ncRNA mediante genotipado, entre los que se encuentran 3 micro RNA (miR) y 2 long non coding RNA (HotAIR), para averiguar si los genes que codifican a estos RNA protegen o predisponen a padecer CM en varones (Tabla 1). A continuación, se analizaron las diferencias entre las distribuciones genotípicas y alélicas de los grupos controles y pacientes de los 5 SNP (Tabla 2).

El estudio del polimorfismo con rs2910164 del gen mir-146A muestra que no existen diferencias en la distribución genotípica entre los pacientes con CM y los controles, pero al realizar el estudio de alelos el polimorfismo con alelo $\mathrm{C}$ parece que reduciría el riesgo de padecer CM.

El estudio del polimorfismo con rs11614913 del gen mir-196A2 no muestra relación entre el desarrollo de $\mathrm{CM}$ en varones y este SNP; la distribución genotípica y de los alelos es similar en ambos grupos.

El estudio del polimorfismo rs3746444 del gen mir-499A muestra que no existen diferencias genotípicas entre pacientes con $\mathrm{CM}$ y controles, la distribución genotípica y de los alelos es similar en ambos grupos. 
TABLA 2. Resultados de los estudios de los distintos ncRNA

\begin{tabular}{|c|c|c|c|c|}
\hline $\begin{array}{l}\text { ncRNA y } \\
\text { polimorfismo }\end{array}$ & Genotipo & Pacientes ( \%) & Controles ( \%) & p-valor* \\
\hline \multirow{9}{*}{$\begin{array}{c}\operatorname{mir}-146 A \\
\text { rs291016 }\end{array}$} & GG & $38(74,5)$ & $42(56,8)$ & \multirow{3}{*}{0,088} \\
\hline & GC & $13(25,5)$ & $30(40,5)$ & \\
\hline & $\mathrm{CC}$ & $0(0,0)$ & $2(2,7)$ & \\
\hline & $G G+G C$ & $51(100,0)$ & $72(97,3)$ & \multirow{2}{*}{0,237} \\
\hline & $\mathrm{CC}$ & $0(0,0)$ & $2(2,7)$ & \\
\hline & $\mathrm{GC}+\mathrm{CC}$ & $13(25,5)$ & $32(43,2)$ & \multirow{2}{*}{0,042} \\
\hline & GG & $38(74,5)$ & $42(56,8)$ & \\
\hline & G & $89(87,3)$ & $114(77,0)$ & \multirow{2}{*}{0,042} \\
\hline & C & $13(12,7)$ & $34(23,0)$ & \\
\hline \multirow{9}{*}{$\begin{array}{l}\text { mir-196A2 } \\
\text { rs11614913 }\end{array}$} & $\mathrm{CC}$ & $17(35,4)$ & $35(42,2)$ & \multirow{3}{*}{0,716} \\
\hline & CT & $23(47,9)$ & $37(44,6)$ & \\
\hline & $\mathrm{TT}$ & $8(16,7)$ & $11(13,3)$ & \\
\hline & $\mathrm{CC}+\mathrm{CT}$ & $40(83,3)$ & $72(86,7)$ & \multirow{2}{*}{0,593} \\
\hline & $\mathrm{TT}$ & $8(16,7)$ & $11(13,3)$ & \\
\hline & $\mathrm{CT}+\mathrm{TT}$ & $31(64,6)$ & $48(57,8)$ & \multirow{2}{*}{0,447} \\
\hline & $\mathrm{CC}$ & $17(35,4)$ & $35(42,2)$ & \\
\hline & C & $57(59,4)$ & $107(64,5)$ & \multirow{2}{*}{0,413} \\
\hline & $\mathrm{T}$ & $39(40,6)$ & $59(35,5)$ & \\
\hline \multirow{9}{*}{$\begin{array}{l}\operatorname{mir}-499 A \\
\text { rs3746444 }\end{array}$} & AA & $27(54,0)$ & $53(66,3)$ & \multirow{3}{*}{0,375} \\
\hline & $A G$ & $19(38,0)$ & $22(27,5)$ & \\
\hline & GG & $4(8,0)$ & $5(6,3)$ & \\
\hline & $\mathrm{AA}+\mathrm{AG}$ & $46(92,0)$ & $75(93,8)$ & \multirow{2}{*}{0,702} \\
\hline & GG & $4(8,0)$ & $5(6,3)$ & \\
\hline & $A G+G G$ & $23(46,0)$ & $27(33,8)$ & \multirow{2}{*}{0,162} \\
\hline & AA & $27(54.0)$ & $53(66,3)$ & \\
\hline & A & $73(73,0)$ & $128(80,0)$ & \multirow{2}{*}{0,19} \\
\hline & G & $27(27,0))$ & $32(20,0)$ & \\
\hline
\end{tabular}


DAVID DÍEZ CASTRO; MARÍA OVEJERO SÁNCHEZ; ROGELIO GONZÁLEZ SARMIENTO ESTUDIO DE NCRNA EN VARONES CON CÁNCER DE MAMA

\begin{tabular}{|c|c|c|c|c|}
\hline $\begin{array}{l}\text { ncRNA y } \\
\text { polimorfismo }\end{array}$ & Genotipo & Pacientes ( \%) & Controles ( \%) & $\mathrm{p}$-valor* \\
\hline \multirow{9}{*}{$\begin{array}{c}\text { HotAIR } \\
\text { rs12826786 }\end{array}$} & $\mathrm{CC}$ & $29(58,0)$ & $23(41,8)$ & \multirow{3}{*}{0,085} \\
\hline & CT & $14(28,0)$ & $27(49,1)$ & \\
\hline & TT & $7(14,0)$ & $5(9,1)$ & \\
\hline & $\mathrm{CC}+\mathrm{CT}$ & $43(86,0)$ & $50(90,9)$ & \multirow{2}{*}{0,43} \\
\hline & $\mathrm{TT}$ & $7(14,0)$ & $5(9,1)$ & \\
\hline & $\mathrm{CT}+\mathrm{TT}$ & $21(42,0)$ & $32(58,2)$ & \multirow{2}{*}{0,098} \\
\hline & $\mathrm{CC}$ & $29(58,0)$ & $23(41,8)$ & \\
\hline & C & $72(72,0)$ & $72(66,4)$ & \multirow{2}{*}{0,378} \\
\hline & $\mathrm{T}$ & $28(28,0)$ & $37(33,6)$ & \\
\hline \multirow{9}{*}{$\begin{array}{l}\text { HotAIR } \\
\text { rs1899663 }\end{array}$} & $\mathrm{CC}$ & $30(61,2)$ & $38(47,5)$ & \multirow{3}{*}{0,046} \\
\hline & CA & $14(28,6)$ & $39(48,8)$ & \\
\hline & AA & $5(10,2)$ & $3(3,8)$ & \\
\hline & $\mathrm{CC}+\mathrm{CA}$ & $44(89,8)$ & $77(96,3)$ & \multirow{2}{*}{0,14} \\
\hline & AA & $5(10,2)$ & $3(3,8)$ & \\
\hline & $\mathrm{CA}+\mathrm{AA}$ & $19(38,8)$ & $42(52,5)$ & \multirow{2}{*}{0,13} \\
\hline & $\mathrm{CC}$ & $30(61,2)$ & $38(47,5)$ & \\
\hline & C & $74(75,5)$ & $115(71,9)$ & \multirow{2}{*}{0,522} \\
\hline & A & $24(24.5)$ & $45(28,1)$ & \\
\hline
\end{tabular}

*En negrita valor estadísticamente significativo $\mathrm{p}<0,05$.

Para el gen HotAIR se estudiaron dos polimorfismos: rs12826786, rs1899663. El primero presenta una distribución genotípica y de los alelos similar entre ambos grupos, por lo que no modifica el riesgo a desarrollar $\mathrm{CM}$ en varones. El segundo polimorfismo, al contrario que en el primero, muestra que el genotipo CA protege frente a la aparición de CM en varones.

\section{Discusión}

En este estudio de casos y controles en varones con cáncer de mama, los datos que hemos obtenido sobre el mir-146A concuerdan con los resultados obtenidos por Hu et al. (Hu et al., 2009) en 2009, los cuales afirman que el alelo C no incrementa el riesgo de padecer $\mathrm{CM}$ en mujeres asiáticas. Asimismo, el estudio de 
Catucci et al. (Catucci et al., 2010) en 2010 también afirma que no hay relación entre el mir-146A y el CM en mujeres de origen italiano y germánico. En cambio, Upadhaya et al. (Upadhaya et al., 2016) realiza el estudio de este mismo ncRNA a un grupo de 160 mujeres caucásico-australianas llegando a otra conclusión, el alelo $\mathrm{C}$ aumenta el riesgo de CM en mujeres. Estas diferencias pueden ser debidas a que el estudio de Upadhaya et al. (Upadhaya et al., 2016) está realizado en un grupo grande de mujeres caucásico-australianas y en el presente trabajo se ha realizado con un grupo de 51 pacientes varones; estas diferencias no solo étnicas sino también de género pueden ser las causantes de que estos dos estudios discrepen.

Respecto a los otros, mir-196A2 y mir-499A, no podemos asociarlos a un aumento del riesgo a padecer CM en varones al igual que el estudio realizado por Catucci et al. (Catucci et al., 2010), aunque esto tampoco se corresponde con el estudio de $\mathrm{Hu}$ et al. (Hu et al., 2009), donde concluyen que la mutación T>C en rs11614913 del miR196A2 y el alelo A ancestral en rs3746444 del miR499A aumentan el riesgo de CM en mujeres chinas. Al igual que un estudio realizado por Dai et al. (Dai et al., 2016) en mujeres chinas, que afirma haber asociaciones entre estos polimorfismos de estos dos últimos ncRNA y el aumento de CM. Al igual que en el caso anterior, estas discrepancias se pueden deber al sexo y la etnia de los pacientes incluidos en los estudios.

En el caso del HotAIR con rs12826786 no existe asociación con un aumento del riesgo de padecer CM en varón, estos resultados son los mismos que Li et al. (Li et al., 2018) en 2018 obtienen en su metaanálisis no solo para CM, sino también para cáncer de cérvix y de ovario. En cambio, en el estudio de Bayram et al. (Bayram et al., 2016), en población femenina turca, el polimorfismo C>T en el rs128126786 está relacionado con un aumento de riesgo de padecer CM, como ocurre en el caso de los miRNA; estas discrepancias pueden ser debidas a la diferencia de género y de etnia.

En el rs1899663 el genotipo CA parece proteger frente a la aparición de CM en varón ya que este genotipo aparece en un número significativamente superior en los controles de la población estudiada. Este resultado no se puede asociar a ninguno de los alelos, cuando hacemos el estudio por separado vemos resultados similares en los dos.

Esta enfermedad es poco prevalente en varones por lo que hay pocos pacientes; esto dificulta el estudio ya que sería necesario tener una población más grande para la realización de un estudio más completo 
DAVID DÍEZ CASTRO; MARÍA OVEJERO SÁNCHEZ; ROGELIO GONZÁLEZ SARMIENTO

ESTUDIO DE NCRNA EN VARONES CON CÁNCER DE MAMA

\section{Conclusiones}

Teniendo en cuenta los resultados, las conclusiones de este trabajo son:

- El alelo C del polimorfismo rs2910164 del gen que codifica el mir-146A podría proteger frente a la aparición de CM en varones.

- El genotipo CA del polimorfismo rs1899663 del gen que codifica HotAIR podría proteger frente a la aparición de CM en varones.

- Los polimorfismos rs11614913 de mir-196A2, rs746444 de mir-499A y rs12826786 de HotAIR no parece que modifiquen el riesgo de desarrollar cáncer de mama en varón en la población incluida en el estudio, pudiendo existir diferencias en función del género y la etnia.

La falta de significación podría deberse a un reducido número de casos, si bien se trata de una enfermedad muy poco frecuente en población masculina.

\section{Bibliografía}

Banco de ADN. Grupo de trabajo de Banco de ADN del instituto de salud Carlos III, PNT extracción de ácidos nucleicos; 2011. pp. 12-13. Disponible en: https://redbiobancos. es/wp-content/uploads/pnt-extraccion-acidos-nucleicos.pdf

Bayram S, Sümbul AT, Dadaş E. A funtional HOTAIR rs12826786 C $>$ T polymorphism is associated with breast cancer susceptibility and poor clinicopathological characteristics in a Turkish population: a hospital-based case-control study. Tumor Biol. 2016; 37(4):5577-5584. https://doi.org/10.1007/s13277-015-4430-y

Catucci I, Yang R, Verderio P, Pizzamiglio S, Heesen L, Hemminki K, Sutter C, Wappenschmidt B, Dick M, Arnold N, Bugert P, Niederacher D, Meindl A, Schmutzler RK, Bartram CC, Ficarazzi F, Tizzoni L, Zaffaroni D, Manoukian S, Barile M, Pierotti MA, Radice P, Burwinkel B, Peterlongo P. Evaluation of SNPs in miR-146a, miR196a2 and miR-499 as low-penetrance alleles in German and Italian familial breast cancer cases. Hum Mutat. 2010; 31(1):E1052-7. https://doi.org/10.1002/humu.21141

Collins LJ, Schönfeld B, Chen XS. Chapter 4 - The epidenetics of non-codingRNA. En: Handbook of epigenetics; 2011. pp. 49-61. DOI: 10.1016/B978-0-12-375709-8.00004-6

Dai ZM, Kang HF, Zhang WG, Li HB, Zhang SQ, Ma XB, Lin S, Wang M, Feng YJ, Liu K, Liu XH, Xu P, Dai ZJ. The Associations of Single Nucleotide Polymorphisms in miR196a2, miR-499, and miR-608 With Breast Cancer Susceptibility: A STROBE-Compliant Observational Study. Medicine (Baltimore). 2016; 95(7):e2826. https://doi.org/10.1097/MD.0000000000002826

Hu Z, Liang J, Wang Z, Tian T, Zhou X, Chen J, Miao R, Wang Y, Wang X, Shen H. Common genetic variants in pre-microRNAs were associated with increased risk of breast cancer in Chinese women. Hum Mutat. 2009; 30(1):79-84. DOI: 10.1002/ humu.20837

Jeffares D, Poole A, Penny D. J Mol Evol. 1998; 46:18. https://doi.org/10.1007/PL00006280 
Li J, Liu R, Tang S, Feng F, Wang X, Qi L, Liu C, Yao Y, Sun C. The effect of long noncoding RNAs HOX transcript antisense intergenic RNA single-nucleotide polymorphisms on breast cancer, cervical cancer, and ovarian cancer susceptibility: A meta-analysis. J Cell Biochem. 2018; 28. https://doi.org/10.1002/jcb.27975.

PATIA ¿En qué consiste el genotipado? [Internet]. Patia diabetes; 8 marzo 2016 [consultado 22 enero 2019]. Disponible en: http://www.patiadiabetes.com/faq-items/ consiste-genotipado/

Peng Y, Croce CM. The role of MicroRNAs in human cancer. Signal Transduct Target Ther. 2016; 28;1:15004. https://doi.org/10.1038/sigtrans.2015.4

Tang Q, Hann SS. HOTAIR: An Oncogenic Long Non-Coding RNA in Human Cancer. Cell Physiol Biochem. 2018; 47:893-913. https://doi.org/10.1159/000490131

Upadhyaya K, Smith RA, Chacon-Cotes D, Revêchon G, Bellis C, Lea RA, Haupt LM, Chambers SK, Youl PH, Griffiths LR. Association of the microRNA-Single Nucleotide Polymorphism rs2910164 in miR146a with sporadic breast cancer susceptibility: A case control study. Gene. 2016; 576:256-260. https://doi.org/10.1016/j.gne.2015.10.019

Vieira, FMJ. Porfiria cutânea tardia com mutações do gene da hemocromatose C282Y e H63D e análise retrospectiva do perfil de ferro em relação ao tratamento: estudo de 60 casos [tesis]. São Paulo: Faculdade de Medicina; 2012 [acceso 2020-1-23]. Disponible en: http://www.teses.usp.br/teses/disponiveis/5/5133/tde-24012013-170707/.

What is epigenetics?, non-coding RNA [Internet]. What is epigenetics? [consultado 26 de enero 2019]. Disponible en: https://www.whatisepigenetics.com/non-coding-rna/

Zhang LH, Hao BB, Zhang CY, Dai XZ, Zhang F. Contributions of polymorphisms in miR146a, miR196a, and miR499 to the development of hepatocellular carcinoma. Genet. Mol. Res. 2016; 15:3. https://doi.org/10.4238/gmr.15038582

Ediciones Universidad de Salamanca / @@ $\quad$ FarmaJournal, vol. 6, núm. 1 (2021), pp. 27-37 
